\title{
AN EFFICIENT APPROACH TO QUERY REFORMULATION IN WEB SEARCH
}

\author{
M. Kiran Kumar ${ }^{1}$, S. Jessica Saritha ${ }^{2}$ \\ ${ }^{1}$ M-Tech, Computer Science \& Engineering, JNTUA College of Engineering Pulivendula, AP, India \\ ${ }^{2}$ Assistant Professor, Computer Science \& Engineering, JNTUA College of Engineering, Pulivendula, AP, India.
}

\begin{abstract}
Wide range of problems regarding to natural language processing, mining of data, bioinformatics and information retrieval can be categorized as string transformation, the following task refers the same. If we give an input string, the system will generates the top $k$ most equivalent output strings which are related to the same input string. In this paper we proposes a narrative and probabilistic method for the transformation of string, which is considered as accurate and also efficient. The approach uses a log linear model, along with the method used for training the model, and also an algorithm that generates the top $k$ outcomes. Log linear method can be defined as restrictive possibility distribution of a result string and the set of rules for the alteration conditioned on key string. It is guaranteed that the resultant top $k$ list will be generated using the algorithm for string generation which is based on pruning. The projected technique is applied to correct the spelling error in query as well as reformulation of queries in case of web based search. Spelling error correction, query reformulation for the related query is not considered in the previous work. Efficiency is not considered as an important issue taken into the consideration in earlier methods and was not focused on improvement of accuracy and efficiency in string transformation. The experimental outcomes on huge scale data show that the projected method is extremely accurate and also efficient.
\end{abstract}

Keywords: Log linear method, Query reformulation, Spelling Error correction. -***.

\section{INTRODUCTION}

This paper focuses on string transformation, which is the most common problem, in various applications. In the processing of natural language correction of spelling errors generation of pronunciations, word stemming can be categorized as string transformation. It can also be used in query reformulation and query implication in search. In the domain of data mining, string transformation is used in synonyms mining and database verification matching. Now days all the application are based on the network kit is compulsory that the transformation must be accurate as well as efficient. If we give an input string, the system will generates the top $\mathrm{k}$ most equivalent output strings which are related to the input string by the usage of many operations. Here any type of tokens such as string of characters, words. Every operator is considered as a rule which defines replacement of substring with its equivalent substring. The possibility of transformation can characterize relationship, importance, and connection between strings in a particular application. Though assured development has been made, supplementary examination of the job is still needed, mainly from the point of view of enhancing accuracy as well as efficiency, which is accurately the objective of this effort. Based on the dictionary usage string transformation can be performed on different settings, on is when the dictionary is used and the other is when not used.. When we use a dictionary, the resultant strings must be present in the dictionary, as the
Volume of the dictionary is very large. Without the simplification, we particularly study about the correction of spelling in query along with the reformulation of the queries in web search. In the initial task, a string contains character set. In the next task, a string is of words. Correcting spelling in queries typically consists of two stages: candidate generation and candidate selection. Candidate generation is used when it is required to identify the most common corrections of misspelled string from dictionary. In such cases, a string consists of characters is considered as input and operators correspond to insert, delete, and substitution with surrounding characters, or without surrounding characters for example, "lly"!"ly". Clearly candidate generation can be considered as an example for string transformation. Note that the candidate generation is disturbed with a solitary word; after candidate creation, the words present in the query can be later engaged to compose the concluding candidate selection.. For example, in case of the abbreviation, while searching the user given the abbreviation as an query in the search interface, but the document in the records contains the full form of that abbreviation, here raises the problem regarding in not identifying the search related records. if we consider "NY Times" as the query and the source document contains "New York Times", in such cases the query and the document does not match fit and then the document cannot be considered as ranked high. Query reformulation attempt to convert "NY Times" into "New York Times" and thus create a better identity between the query and the document. Earlier effort on string transformation is categorized into two groups. Few work groups mainly consider efficient 
generation of strings. The other effort tried to find out the model with dissimilar approaches, such as a generative method, a logistic regression method, and the discriminative model. Yet, efficiency is not considered as an important factor in these methods.

\section{LITERATURE SURVEY}

It is an important factor for a browser to check the spelling of the query term entered in the search interface to make the search efficient and to get the results in the efficient manner. This paper describes narrative methods for the use of distributional similarity estimated from query logs in learning improved query spelling correction models. The key point in our methods is to measure the distributional similarities between the two terms. When measured it is known to be high between the frequently occurring mistakes in spellings and those correction, and it is found to be low two immaterial terms only with same spellings.

According to winnow-based approach for context-sensitive correction of spellings, for a large variety of problems there is a requirement of characterization of linguistics. It focus on the concepts of techniques that are used for correcting the strings errors in of miss-placed so as to avoid these kinds of problems while searching on the net the earlier techniques used the winnow based approaches for correcting those errors. In this effort we are acquiring the properties of those methods to avoid such mistakes. if we consider the example string " to", suppose if we place the string "too" in place of "to" it leads to difference in meanings and may the sentence leads to wrong. This is the task of fixing spelling errors that happen to result in valid words, such as substituting to for too, casual for causal, and so on.

A unified and discriminative method for query modification is a method used for providing the alternative substrings in place of original string, in the case when the original string is found to be misspelled.

\section{FUNDAMENTALS}

\subsection{Spelling Error Correction}

In this module if a user wants to check the spelling, He/She can check it and correct it automatically. Efficiency is critical for this job due to following reasons.

(1) The dictionary is very large and (2) The response time must be very short.

The initial point indicates, while using the dictionary it is very difficult to find the required string if in case it is misspelled when the size of the dictionary is very large. The second point indicates that the response time is based on the size of the dictionary present. Large the size of the dictionary more will the response time and small the size of the dictionary low will be the response time.

\subsubsection{Word Pair Mining}

Searching on web by a user is of session based. That session will be of the frequently made mistakes and the spelling errors in the query term. In order to avoid those mistakes pairing of strings must be done in the browser which indicates that the string which is spelt wrong must be paired with the correct spelling of the same word. This leads to replacement of the wrong spelt word with the correct spelling. The following are some of the examples of the word pairs with misspelled and correct spelled.

Table-1: Examples of Word Pairs

\begin{tabular}{|l|l|l|l|}
\hline Misspelled & Correct & Misspelled & Correct \\
\hline Aacoustic & Acoustic & Chevorle & Chevrolet \\
\hline Liyerature & Literature & Tournemen & Tournament \\
\hline Shingle & Shingle & Newpape & Newspaper \\
\hline Finlad & Finland & Ccomponet & Component \\
\hline Reteive & Retrieve & Olimpick & Olympic \\
\hline
\end{tabular}

\subsection{String Transformation}

Here we are using two techniques for searching the String 1)String Generation 2)String Transformation.

String Generation: Here we have to generate 50,000 Strings in the alphabetical order. Starting from a to $\mathrm{z}$ like a,aa,.....z. Generating the strings manually takes large amount of time and it is recommended to use the database with thousands of words, by connecting the database to the required system.

String Transformation: It means we have given the user with the advantage of the String Generation along with the String alias. For example if the end user have typed "TKDE" which is equal to "Transactions on Knowledge and Data Engineering", the search interface may be able to find the related result.

String Mining: The User can be able to download the string along with its synonyms and also he can be able to download its related substrings and its inverse etc. The user can also check whether the given string is present in the collection of strings, if it is present in the group the result will be "String is Found" and if string is not present the result will be "String is Not Found".

\section{EXISTING \& PROPOSED SYSTEMS}

Earlier work on string transformation is of two categories the initial works mainly focused on the methods that are employed for the generation of stings in efficient manner. The later works tried to develop the models with various types of approaches. Spelling error correction, query reformulation and synonym mining for the related query are not taken into consideration in the earlier work. In the previous methods and techniques they are not considered the efficiency and accuracy as the important factors. A loglinear method for string transformation with the use of efficient method for generating string is used in the present work. Two specific Applications are associated with our method namely

1. Spelling error Correction of Queries.

2. Query Reformulation in web search. 
As the proposed system is focused on the log-linear model for string Transformation Along with the spelling error check and query reformulation in web search, the efficiency and accuracy can be enhanced. Query reformulation helps the user in generating the related substrings in the top $\mathrm{k}$ candidate list.

\subsection{Model for String Transformation}

Here we are proposing a alignment procedure which is known as the edit distance based alignment.

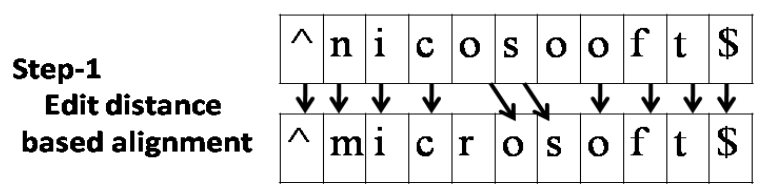

\section{Step-2 \\ Derived rule $\quad N \rightarrow m, 0 \rightarrow r, 0->0$}

Fig-2 Edit distance based alignment

It consists of three steps. In the initial step the query is represented in edit distance style. In the second step we derive a rule by which the representation is done in the previous step. We expand the rules with context in the last step. The representation is as follows.

\subsection{Architecture}

\section{Architecture}

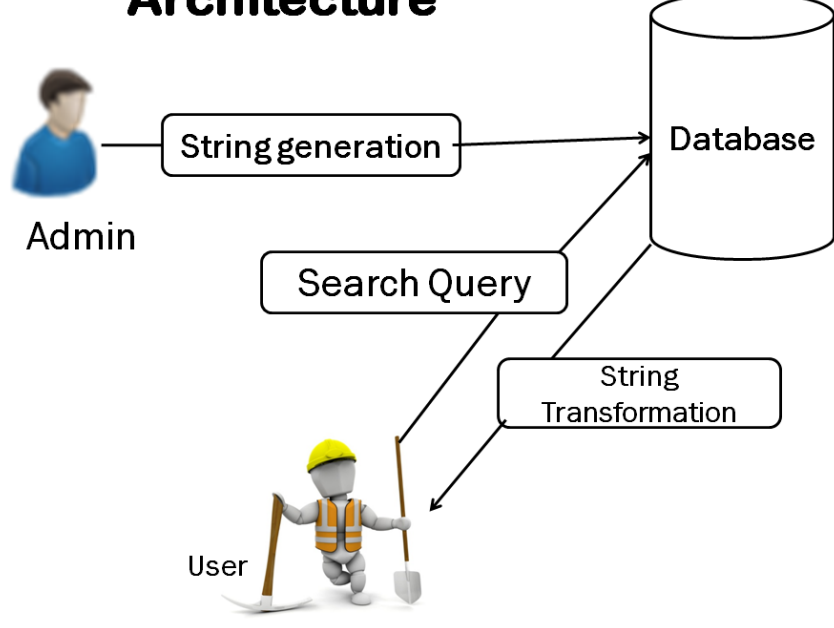

Fig 1 Architecture of proposed system

The above figure indicates the proposed architecture in which the database, admin and the end user involves. In the string generation phase the strings are generated by the admin either by manually or the database with huge amount of data is connected. When the user want to perform the operation such as retrieving the data from the web, regarding to the query the transformation process is done at the browser in order to rectify any mistakes regarding to spelling etc. the query reformulation is done whine receiving the query from the user at the search interface.

\section{CONCLUSION}

In this paper, we have projected new statistical learning methods for string transformation. Proposed method is narrative and distinctive in its sculpt, learning algorithm, and the algorithm of string generation. Two detailed applications are associated with our technique, they are spelling error rectification in queries and the query reformulation during web search. Experimental consequences on two huge data sets and Microsoft Speller shows that proposed method improves on the baselines in case of accurateness and effectiveness. Our proposed method is mainly useful when the problem occurs on a outsized scale.

In case of large scale systems our method of query reformulation is well used because it is very easy to retrieve the information regarding to the search query even in the case of substitutes. Our proposed log linear model can be used in these system for efficient results.

\section{REFERENCES}

[1]. M. Li, M. Zhu, Y. Zhang and M. Zhou, "Exploring distributional likeness based methods for query spelling error correction".

[2]. D. Roth, R. A. Golding "A winnow-based method to context-sensitive correction".

[3]. J. Gauo, H. Li, and X. Cheng, G. Xu, "A combined and discriminative model for query alteration," in Proceedings in the 31st annual intercontinental ACM SIGIR confernce on Research and devlopment in information recovery.

[4]. A. Bem, C. Li, and J. Lu, S. Ji, "Space-constrained gram-based indexing for well-organized rough string search," Proceedings in the 2009 IEEE International Confernce on Data Mining Engineering.

[5]. E. Brill and R. C. Moore, "An improved error model for noisy channel spelling correction," in Proceedings of the 38th Annual Meeting on Association for Computational Linguistics.

[6]. G. Xu and J. Xu, "Learning similarity function for rare queries," in Proc. 4th ACM Int. Conf. Web Search and Data Mining, NewYork.

[7]. C. A. Knoblock, S. Tejada, "Learning province selfsufficient string transformation in weights for high accurateness object classification," in Proc. ACM SIGKDD Int. Conf. Knowledge and Data Mining.

[8]. A. Arasu, S. Chaudhuri, and R. Kaushik, "Learning transformations through examples," Proc. VLDB Endow., vol. 2, pp. 514-525, 2009.

[9]. S. Tejada, C. Knoblock, and S. Minton, "Learning domainindependent string transformation for high accuracy identification," Proc. 8th ACM SIGKDD Int. Conf. Knowledge and Data Mining, New York, USA, 2002.

[10]. C. Li, "Efficient estimated search for string collections," VLDB Endow., vol. 3, no. 2,. 1660-1661

[11]. C. Li, B. Wang, and X. Yang, Improving presentation of estimated queries on thread collections using variablelength ," Proc. 33rd Int. Conf. Very Large Data Bases, Vienna, 
[12]. X. Yang, C. Li, B. Wang, "Cost-based variable-lengthgram collection for string collections to hold up estimated queries competently," in Proc. ACM SIGMOD Int. Conf. Data Mining, New York, USA, pp. 353-364.

\section{BIOGRAPHIES}

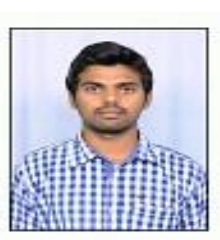

M . Kiran kumar, M-Tech, Department of CSE, JNTUA college of engineering, Pulivendula, Andhra Pradesh, India.

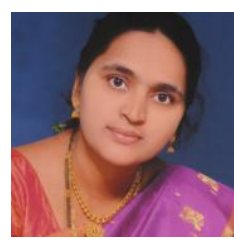

Smt. S.Jessica Saritha is currently working as an Assistant Professor in Department of CSE, JNTUA College of Engineering, Pulivendula Andhra Pradesh India Her Research interests are Data mining and diustributed computing. 